\title{
Clinical Outcomes and Adverse Events of the Stomach Endoscopic Submucosal Dissection of the Mid to Upper Stomach Under General Anesthesia and Monitored Anesthetic Care
}

Jong-In Chang

Samsung Medical Center

Tae Jun Kim

Samsung Medical Center

Na Young Hwang

Samsung Medical Center

Insuk Sohn

Samsung Medical Center

Yang Won Min

Samsung Medical Center

Hyuk Lee

Samsung Medical Center

Byung-Hoon Min

Samsung Medical Center

Jun Haeng Lee

Samsung Medical Center

Poong-Lyul Rhee

Samsung Medical Center

JAE KIM ( $\nabla_{\text {jjkimgm@gmail.com ) }}$

Department of Medicine, Samsung Medical Center, Sungkyunkwan University School of Medicine, Seoul, Korea

Research article

Keywords: endoscopic submucosal dissection, early gastric cancer, anesthesia

Posted Date: August 27th, 2020

DOl: https://doi.org/10.21203/rs.3.rs-61803/v1 
License: (c) (i) This work is licensed under a Creative Commons Attribution 4.0 International License. Read Full License 


\section{Abstract}

Background: Endoscopic submucosal dissection (ESD) of gastric tumors in the mid to upper stomach is a technically challenging procedure. This study compared the therapeutic outcomes and adverse events following ESD of tumors in this area, performed in conjunction with either general anesthesia (GA) or monitored anesthetic care (MAC).

Methods: Between 2012 and 2018, 674 patients underwent ESD of gastric tumors in the midbody, high body, fundus, or cardia (100 patients received GA; 574 received MAC). The results were analyzed using propensity score (PS)-matched (1:1) patients receiving either GA or MAC.

Results: PS matching identified 95 patients who received GA and 95 matched patients who received MAC. Both groups showed high rates of en-bloc resection (GA, 98.5\%; MAC, 98.9\%; $p=0.18$ ) and complete resection, defined as tumors excised with histologically confirmed negative margins (GA, 82.1\%; MAC, $90.5 \% ; p=0.14)$. There were no significant differences in the frequencies of adverse events (GA, $16.8 \%$; MAC, $9.5 \% ; p=0.13$ ) between the anesthetic groups. A logistic regression analysis indicated that the anesthetic method was not a factor impacting the frequencies of complete resection or adverse events.

Conclusion: At our high-volume center, good therapeutic outcomes were achieved following ESD of tumors in the mid to upper stomach, regardless of the anesthetic method used. Our results demonstrate the efficacy and safety non-inferiority of the ESD procedure performed in conjunction with MAC, compared with GA.

\section{Background}

Presently, endoscopic submucosal dissection (ESD) is widely used because it can achieve a higher rate of complete gastric tumor resection than endoscopic mucosal resection [1,2]. Additionally, ESD is less invasive and can result in a better patient quality of life than surgical gastrectomy, if indicated [3]. However, ESD requires sophisticated endoscopic techniques. Thus, the adverse events, such as bleeding and perforations, and clinical outcomes following en-bloc resection are related to the technical skill of the endoscopists, especially in difficult ESD cases. A previous study demonstrated that tumors located in the upper third of the stomach are associated with longer procedure times and higher frequencies of incomplete resection than those located in other stomach areas [3]. Other studies also showed that the mid to upper stomach region has more vessels and is more likely to demonstrate early gastric cancers (EGCs) with submucosal invasion than other areas of the stomach $[4,5]$. Therefore, ESD procedures for tumors located in the mid to upper stomach require high levels of endoscopic technical skill.

ESD also requires minimal patient movement during the procedure [6, 7]. Therefore, previous studies have discussed appropriate sedation methods for use during ESD. Specifically, these studies have compared different sedatives, such as midazolam and propofol, or have compared traditional sedation (delivered by endoscopists) with sedatives and anesthetic care delivered via intubation [1, 2, 6-9]. Some studies have also reported ESD outcomes involving patients under general anesthesia $(G A)[1,10]$. 
The present study was based on the premise that ESD involving the mid to upper stomach is more difficult than that involving the lower part of the stomach. Hence, we assumed that the resection in the mid to upper stomach are less likely to be complete and are more likely to experience adverse events than those in the lower stomach occur than the other. ESD conducted under GA, with intubation, are recognized to be more effective and safer than procedures involving traditional sedation delivered by endoscopists $[1,2,10]$. However, a direct comparison between monitored anesthetic care (MAC), involving mask ventilation, and GA has not been previously reported. Thus, this study compared the therapeutic outcomes and adverse event rates following ESD of tumors located in the mid to upper stomach under either GA or MAC.

\section{Methods}

\section{Study design, setting, and participants}

This retrospective study was performed at Samsung Medical Center, Seoul, South Korea. Between January 2012 and December 2018, 3760 patients underwent ESD of gastric tumors involving either GA or MAC. The tumors were located in the mid to upper stomach, including in the midbody, high body, fundus, and cardia. Histologically confirmed EGCs, adenomas, and neuroendocrine tumors (NETs) were included, based on the final pathologic reports from the ESD specimens (Fig. 1). Patients with prior histories of esophagectomies for esophageal cancer were excluded. The study protocol was reviewed and approved by the institutional review board at Samsung Medical Center. Because this study was based on a retrospective analysis of existing clinical data, the requirement for informed patient consent was waived by the institutional review board (No. 2019-09-015-001).

\section{ESD procedure}

Five gastroenterologists performed all the gastric ESD procedures using a standard technique. First, a circumferential mark was made around the lesion with either a needle knife or a dual knife. Thereafter, fluid (normal saline [100 mL], epinephrine [1 mL], and $0.8 \%$ indigo carmine [0.1 mL]) was injected into the submucosal layer, a circumferential mucosal precut was made, and the submucosal layer was dissected using various types of knives, such as the IT2 knife or dual knife. Endoscopic hemostasis was performed simultaneously whenever bleeding was observed (Fig. 2).

\section{Anesthesia}

The ESD procedure was performed under either GA, with endotracheal intubation, or MAC, with mask ventilation. GA was induced using rocuronium, remifentanil, and propofol, and was maintained using propofol and remifentanil. MAC was induced using midazolam and was maintained using propofol and remifentanil. During anesthesia, the patients were monitored using end-tidal carbon dioxide level, tidal volume, respiratory rate, oxygen saturation, electrocardiogram, and non-invasive blood pressure, body temperature, and heart rate. 


\section{Study outcomes, variables, and definitions}

Data in this study were retrospectively obtained from the electronic medical records of Samsung Medical Center. We collected data regarding patient demographics (age, sex, past medical history, and body mass index), tumor characteristics (tumor location, endoscopic tumor morphology, gross and pathologic tumor size, tumor specimen size, and tumor histopathology), and procedure-related factors (procedure time, anesthetic time, sedation method, and adverse events). The details of the pathologic findings, such as depth of invasion, tumor lateral and vertical margins, lymphatic and vascular involvement, and presence of ulceration, were obtained for EGC cases.

The study's primary outcomes were the frequencies of en-bloc and complete resection. The secondary outcomes were the associated adverse event (bleeding and perforations) frequencies. En-bloc resection was defined as tumor resection as a single piece. Complete resection was defined as en-bloc tumor resection with histologically confirmed tumor-free resection margins, both laterally and vertically. Curative resection, defined as complete resection without submucosal invasion deeper than $500 \mu \mathrm{m}$, lymphatic invasion, or vascular involvement, was assessed in EGC cases. We also compared procedure times, defined as the time from the first observation of the lesion to complete lesion removal, including hemostasis, for the two anesthetic method groups. A perforation was defined as evidence of free air in the radiographic findings, after finishing the ESD procedure. ESD-related bleeding was defined as the need for hemostatic procedures after the ESD procedure.

\section{Statistical analysis}

We used propensity score (PS) matched (1:1) analysis for patients who underwent GA with those who underwent MAC. For patients with EGCs, adenomas, and NETs, the PS matching involved the tumor locations and specimen sizes. In patients with EGCs, PS matching also included the depth of tumor invasion.

Baseline characteristics are summarized as means \pm standard deviation (SD) or as frequencies (percent). Categorical variables were compared using the Chi-square test or Fisher exact test, and continuous variables were compared using Student's $t$-test. Logistic regression was performed and the odds ratio was calculated to identify factors associated with therapeutic outcomes and adverse events; a $p$-value $<0.05$ was considered statistically significant. Statistical analyses were performed using both SPSS, version 25.0 (SPSS, Chicago, IL, USA), and R, version 3.6.1 (R Foundation, Vienna, Austria).

\section{Results}

\section{Clinicopathologic characteristics and clinical outcomes}

The clinicopathologic characteristics of the analyzed patients with EGCs, adenomas, and NETs are shown in Table 1. PSs were used to match 95 patients who underwent GA with 95 who underwent MAC. The patients undergoing the two different anesthetic methods displayed similar clinicopathologic 
characteristics, except for sex and numbers of lesions. Patients undergoing GA (20\%) were more likely to have multiple lesions compared with those undergoing $\operatorname{MAC}(7.4 \%, p=0.03)$. 
Table 1

Clinicopathologic characteristics of EGC, adenoma, NET patients

\begin{tabular}{|c|c|c|c|}
\hline & $\mathrm{GA}(n=95)$ & $\operatorname{MAC}(n=95)$ & $p$ value \\
\hline Age at procedure (years) & $64.9 \pm 11.2$ & $66.5 \pm 9.4$ & 0.28 \\
\hline Sex (male) & $84(88.4 \%)$ & $64(67.4 \%)$ & $<0.001$ \\
\hline BMI (kg/m2) & $24.4 \pm 3.3$ & $24.2 \pm 3.1$ & 0.64 \\
\hline Smoking & & & 0.19 \\
\hline Never & $39(41.0 \%)$ & $50(52.6 \%)$ & \\
\hline Ex & $43(45.3 \%)$ & $31(32.6 \%)$ & \\
\hline Current & $13(13.7 \%)$ & $14(14.8 \%)$ & \\
\hline Pre-operative comorbidities ${ }^{\star}$ (yes) & $51(53.7 \%)$ & $56(52.3 \%)$ & 0.47 \\
\hline Gastrectomy before ESD (yes) & $8(8.4 \%)$ & $5(5.3 \%)$ & 0.39 \\
\hline Number of Lesion & & & 0.03 \\
\hline 1 & $76(80.0 \%)$ & $88(92.6 \%)$ & \\
\hline 2 & $16(16.8 \%)$ & $7(7.4 \%)$ & \\
\hline 3 & $2(2.1 \%)$ & $0(0.0 \%)$ & \\
\hline 4 & $1(1.1 \%)$ & $0(0.0 \%)$ & \\
\hline Location (\%) & & & 0.99 \\
\hline Mid body & $22(23.2)$ & $22(23.2)$ & \\
\hline High body + Fundus & $31(32.6)$ & $30(31.6)$ & \\
\hline Cardia + EG junction & $42(44.2)$ & $43(45.3)$ & \\
\hline Pathology of tumor & & & 0.02 \\
\hline EGC & $81(85.3 \%)$ & $69(72.6 \%)$ & \\
\hline Adenoma & $11(11.6 \%)$ & $25(26.3 \%)$ & \\
\hline Neuroendocrine tumor & $3(3.1 \%)$ & $1(1.1 \%)$ & \\
\hline Morphology of tumor & & & 0.24 \\
\hline
\end{tabular}

$E S D$ endoscopic submucosal dissection, $G A$ general anesthesia, $M A C$ monitored anesthetic care, $E G C$ early gastric cancer, NET neuroendocrine tumor

*Pre-operative comorbidities: One of hypertension, diabetes mellitus, cardiovascular disease, arrhythmia, cerebrovascular disease, chronic pulmonary obstructive disease, chronic liver disease, chronic kidney disease, other malignancies 


\begin{tabular}{|llll|}
\hline & GA $(n=95)$ & MAC $(n=95)$ & p value \\
\hline Elevated & $60(63.2 \%)$ & $48(50.5 \%)$ & \\
\hline Flat & $8(8.4 \%)$ & $9(9.5 \%)$ & \\
\hline Depressed & $27(28.4 \%)$ & $37(38.9 \%)$ & \\
\hline Endoscopic tumor size (cm) & $2.02 \pm 1.22$ & $1.89 \pm 1.13$ & 0.44 \\
\hline Pathologic tumor size $(\mathrm{cm})$ & $2.39 \pm 2.59$ & $1.88 \pm 1.24$ & 0.08 \\
\hline Specimen size $(\mathrm{cm})$ & $4.82 \pm 1.51$ & $4.72 \pm 1.38$ & 0.63 \\
\hline $\begin{array}{l}\text { ESD endoscopic submucosal dissection, GA general anesthesia, MAC monitored anesthetic care, EGC } \\
\text { early gastric cancer, NET neuroendocrine tumor }\end{array}$ & & \\
\hline $\begin{array}{l}\text { *Pre-operative comorbidities: One of hypertension, diabetes mellitus, cardiovascular disease, } \\
\text { arrhythmia, cerebrovascular disease, chronic pulmonary obstructive disease, chronic liver disease, } \\
\text { chronic kidney disease, other malignancies }\end{array}$ & \\
\hline
\end{tabular}

Supplementary Table 1 shows the clinicopathologic characteristics of the PS-matched EGC patients. In this analysis, the patients undergoing GA $(19.5 \%)$ were also more likely to have multiple lesions compared with those undergoing MAC $(3.9 \%, p=0.005)$. The tumor morphologies and histologic differentiations were not significantly different between the two groups.

We compared the clinical outcomes between the two anesthesia groups of matched patients with EGCs, adenomas, and NETs. The procedure time per lesion was longer in the GA group than in the MAC group ( $p$ $<0.001$ ) and the mean minimum resection margin was shorter in the GA group than in the MAC group ( $p$ $=0.04)$. Both anesthetic groups had high rates of en-bloc (GA, 98.5\%; MAC, 98.9\%) and complete (GA, $82.1 \%$; MAC, $90.5 \%)$ resection. There were no significant between-group differences $(p=0.13)$ in the frequencies of adverse events (perforations and bleeding) (Table 2). 
Table 2

Clinical outcomes and adverse events of EGC, adenoma, NET patients

\begin{tabular}{|llll|}
\hline & GA $(n=95)$ & MAC $(n=95)$ & $p$ value \\
\hline En-bloc resection (yes) & $91(98.5 \%)$ & $94(98.9 \%)$ & 0.18 \\
\hline Complete resection (yes) & $78(82.1 \%)$ & $86(90.5 \%)$ & 0.14 \\
\hline Resection margin (minimum, mm) & $4.91 \pm 3.31$ & $6.09 \pm 3.58$ & 0.04 \\
\hline Anesthetic time, total (min) & $131.6 \pm 59.6$ & $78.8 \pm 44.9$ & $<0.001$ \\
\hline Procedure time per, total (min) & $105.5 \pm 59.9$ & $70.5 \pm 44.4$ & $<0.001$ \\
\hline Procedure time per 1 lesion (min) & $91.7 \pm 58.3$ & $66.4 \pm 44.4$ & 0.001 \\
\hline Adverse events (yes) & $16(16.8 \%)$ & $9(9.5 \%)$ & 0.13 \\
\hline Perforation (yes) & $12(12.6 \%)$ & $5(5.3 \%)$ & 0.08 \\
\hline Bleeding (yes) & $5(5.3 \%)$ & $4(4.5 \%)$ & 1.00 \\
\hline $\begin{array}{l}\text { GA general anesthesia, MAC monitored anesthetic care, EGC early gastric cancer, NET neuroendocrine } \\
\text { tumor }\end{array}$ & & \\
\hline
\end{tabular}

The matched EGC patients showed similar results. The rates of en-bloc (GA, 94.8\%; MAC, 98.7\%) and complete (GA, 79.2\%; MAC, 84.4\%) resection were high in both groups. However, the curative resection rates were lower than the complete resection rates, although the differences were not significant, due to the more severe tumor characteristics, such as tumor submucosal invasion $>500 \mu \mathrm{m}$, lymphatic invasion, or vascular involvement (GA, 61.0\%; MAC, 70.1\%) (Supplementary Table 2).

\section{Association between therapeutic outcome and anesthetic modality}

In the multivariable analysis, the anesthetic method was not a factor associated with complete ESD resection of gastric tumors (Table 3, Supplementary Table 3); a long resection margin was the only factor associated with complete resection of gastric tumors (Table 3). In the regression analysis, the anesthetic method was also not associated with the adverse event rate (Table 4, Supplementary Table 4). 
Table 3

Factors associated with complete resection in EGC, adenoma, NET patients

\begin{tabular}{|c|c|c|c|c|}
\hline & \multicolumn{2}{|l|}{ Univariate Analysis } & \multicolumn{2}{|c|}{ Multivariate Analysis } \\
\hline & OR $(95 \% \mathrm{Cl})$ & $p$ value & OR $(95 \% \mathrm{Cl})$ & $p$ value \\
\hline Age (years) & $0.93(0.96-1.04)$ & 0.99 & & \\
\hline Male (vs. female) & $1.07(0.40-2.86)$ & 0.90 & & \\
\hline \multicolumn{5}{|l|}{ Methods of anesthesia } \\
\hline GA & 1.00 (reference) & & 1.00 (reference) & \\
\hline MAC & $2.08(0.88-4.94)$ & 0.09 & $0.98(0.30-3.18)$ & 0.98 \\
\hline Number of lesion $\geq 2$ (vs. 1 ) & $0.62(0.21-1.81)$ & 0.38 & & \\
\hline Endoscopic tumor size (cm) & $0.72(0.54-0.98)$ & 0.03 & $0.49(0.22-1.12)$ & 0.09 \\
\hline Pathologic tumor size (cm) & $0.83(0.67-1.03)$ & 0.09 & $0.96(0.75-1.22)$ & 0.72 \\
\hline Specimen size (cm) & $0.82(0.63-1.07)$ & 0.14 & $1.26(0.63-1.12)$ & 0.52 \\
\hline $\begin{array}{l}\text { Resection margin } \\
\text { (minimum, mm) }\end{array}$ & $1.70(1.34-2.15)$ & $<0.001$ & $1.77(1.35-2.33)$ & $<0.001$ \\
\hline Procedure time per 1 lesion (min) & $0.99(0.98-0.99)$ & 0.002 & $0.99(0.98-1.01)$ & 0.14 \\
\hline \multicolumn{5}{|l|}{ Pathology of tumor } \\
\hline EGC & 1.00 (reference) & - & & \\
\hline Adenoma + NET & $0.28(0.06-1.22)$ & 0.28 & & \\
\hline
\end{tabular}


Table 4

Factors associated with adverse events in EGC, adenoma, NET patients

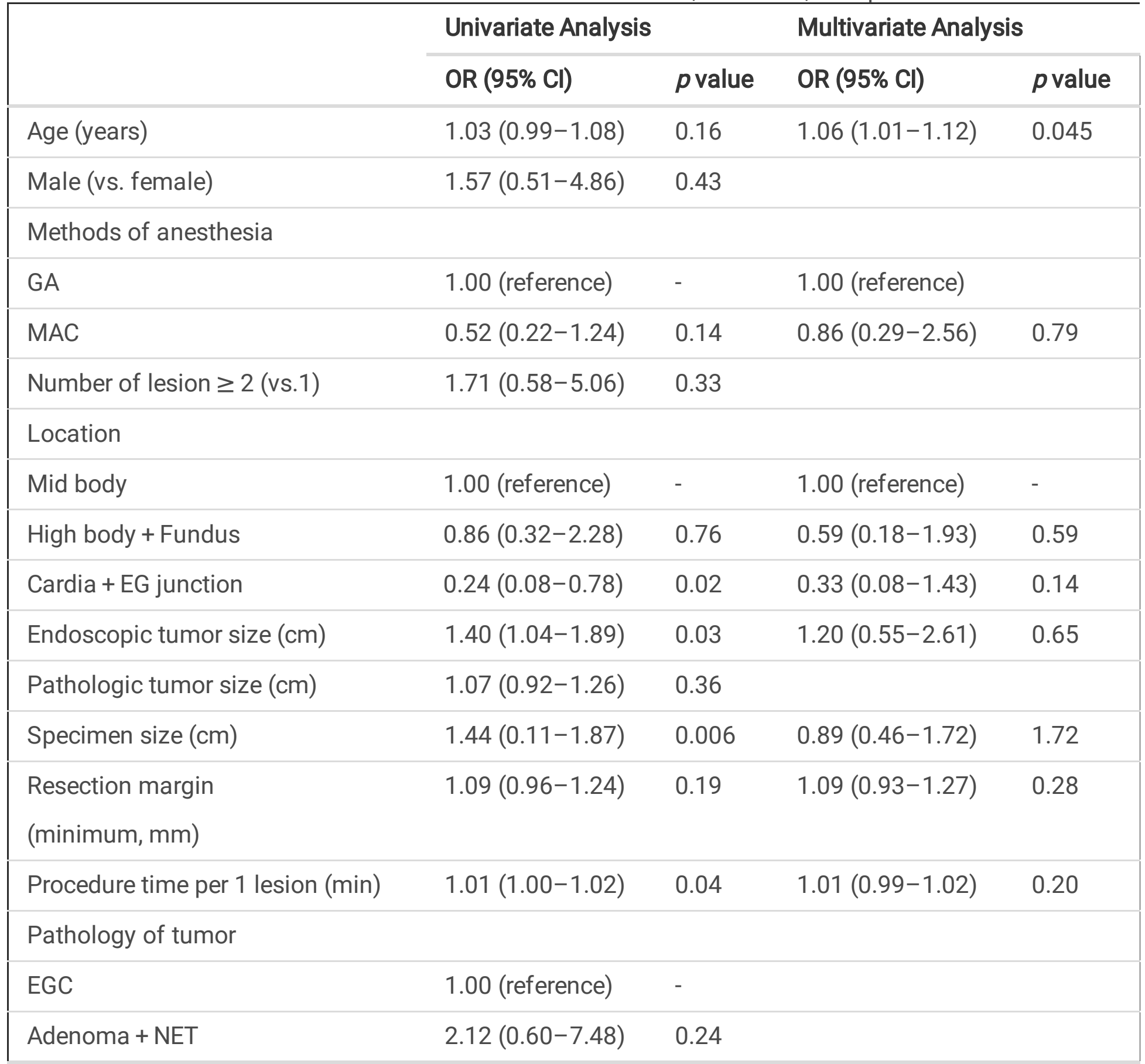

$G A$ general anesthesia, $M A C$ monitored anesthetic care, $E G$ esophagogastric, $E G C$ early gastric cancer, $N E T$ neuroendocrine tumor, $O R$ odds ratio, $C /$ confidence interval

\section{Discussion}

ESD is widely used to treat gastric tumors and is often performed under either anesthesia or traditional sedation, administered by endoscopists $[1,8,10]$. Previous studies have compared outcomes based on the sedatives or anesthetic methods used. However, there have been insufficient data comparing the anesthetic methods (GA and MAC) used during difficult gastric ESD cases. To the best our knowledge, 
this is the first study comparing the therapeutic outcomes and adverse events following ESD of tumors, performed under GA or MAC, in the upper region of the stomach.

In this present study, the en-bloc resection rates were $>98 \%$ for both anesthetic groups and the complete resection rates were $>82 \%$; similar results were observed when the EGC cases were separately examined. In a previous meta-analysis of ESD clinical outcomes, the en-bloc resection rate for 1437 cases of EGC was $92.4 \%$ and the complete resection rate for 1495 cases of EGC was $82.4 \%$, regardless of the anesthetic method or tumor location [11]. Thus, the en-bloc and complete resection rates in our study were above average. However, our curative resection rates, for EGC cases, were relatively low (GA, 61.0\%; MAC, 70.1\%). A previous study of post-ESD, short-term outcomes, involving 712 patients in a prospective multicenter cohort study in Korea, showed an en-bloc resection rate of $97.3 \%$ and a curative resection rate of $86.8 \%$. The authors of that study suggested that non-curative resection were associated with large lesions, submucosal invasion, and moderately or poorly differentiated adenocarcinomas [12]. Also in that study, $26.1 \%$ of the patients had EGC lesions $>2 \mathrm{~cm}$ in size, $33.1 \%$ of the lesions were moderately or poorly differentiated adenocarcinomas, and $16.0 \%$ of the lesions had invaded the submucosal layer [12]. In our study, $35.1 \%$ of EGCs were $>2 \mathrm{~cm}$ in size, $63.6 \%$ were moderately or poorly differentiated adenocarcinomas, and $44.8 \%$ showed submucosal invasion. Thus, the lower curative resection rate, in our study, may have been due to the tumor characteristics of our study population.

In the present study, the rates of ESD-related perforations were $12.6 \%$ for patients receiving GA and $9.5 \%$ for those receiving MAC; the respective bleeding rates were $5.3 \%$ and $4.5 \%$. Amongst EGC cases, the perforation rates were $13.0 \%$ for those receiving GA and $5.2 \%$ for those receiving MAC; the bleeding rates were $5.2 \%$ and $3.9 \%$, respectively. In a previous meta-analysis of ESD adverse events, a perforation rate of $4.3 \%$ was observed among 1437 EGC cases and bleeding occurred in $9.4 \%$ of 876 EGC cases, regardless of the anesthetic method or tumor location [11]. The higher incidence of perforations, in our study, may also be related to the tumor characteristics of our study population, as described for the curative resection rates. ESD involving the upper part of the stomach is carries a higher risk of adverse events, such as perforations and bleeding, due to the difficulty of positioning the ESD knife, the relatively thin gastric wall, and the associated vasculature; therefore, the procedure requires longer procedure times and more advanced technical skills than procedures involving the lower portion of the stomach $[4,13,14]$. Moreover, EGC submucosal invasion occurs more frequently in the mid and upper parts of the stomach [5]. A previous study of the risk factors for procedure-related perforations demonstrated that longer procedure times are an associated risk factor. That study also showed that the perforation rate was higher when the upper stomach was involved ( $8 \%$ of 478 cases) than when the lower stomach was involved $(0.5 \%$ of 478 cases) [15]. Another study showed that the perforation risk is 4.9-fold higher for procedures involving the upper stomach than for those involving other areas of the stomach, after adjusting for submucosal invasion and dyslipidemia [16]. In the present study, the procedure times were longer for patients receiving GA than for those receiving MAC. However, the perforation rates were not significantly different between the two anesthetic groups. The results show that the two anesthetic methods are not associated with ESD clinical outcomes or adverse events; thus, safe and efficacious ESD may be achieved using MAC in highvolume centers with specialized endoscopists. 
To develop the hypothesis for our study, we presumed that ESD performed under GA would have better therapeutic outcomes and fewer adverse events than those not performed under GA, because GA would prevent patients from demonstrating even subtle movements during the procedure. As noted previously, previous study results involving esophageal or gastric ESD indicated that GA decreases the risk of adverse events, compared with traditional sedation administered by endoscopists [2, 17]. Further, a previous study of esophageal ESD procedures, conducted at our institute, showed that the en-bloc resection rate was significantly higher and perforation rate was significantly lower in patients receiving GA than in those receiving traditional sedation; GA was shown to be a factor associated with achieving complete resection and minimizing perforations [17]. Despite only a few studies comparing GA and MAC, MAC has been shown to provide more clinical benefits than GA. One retrospective study compared endovascular angioplasty outcomes, in patients with aortoiliac disease, according to the intra-operative use of GA or MAC. Interestingly, the post-operative adverse event rate was significantly lower for procedures performed using MAC than for those using GA [18]. Similarly, another retrospective study also suggested that MAC is a safe anesthetic method for mid-gestation pregnant women and its use is associated with a lower adverse event rate than GA [18].

Our study has limitations due to its retrospective design and its inclusion of procedures performed only at one medical center. However, as a high-volume center that employs several technically advanced endoscopists, many stomach ESD cases have been performed and were available for inclusion. Since 2012, when ESD was introduced at our center, most cases have utilized traditional sedation or MAC. However, a selection bias exists because endoscopists request specific anesthetic methods, before starting their procedures. Endoscopists tend to request GA for difficult cases, such as those involving tumors in the upper stomach or that are presumed to be more invasive, based on morphologic assessments. Moreover, procedures involving the mid to upper stomach are 5 times more likely to involve MAC than GA. To overcome this limitation, we applied PS matching.

In conclusion, our study results demonstrated that good clinical outcomes were achieved following ESD of tumors in the mid to upper stomach, regardless of the anesthetic method used in our high-volume center. Further, the results showed the non-inferiority of the safety and therapeutic outcomes following ESD procedures performed in conjunction with MAC, compared with those performed with GA. Regarding cost-effectiveness and less invasiveness, gastric ESD under MAC might be superior to ESD under GA.

\section{Abbreviations}

used in this paper: ESD, endoscopic submucosal dissection; GA, general anesthesia; MAC, monitored anesthetic care; PS, propensity score; EMR, endoscopic mucosal resection; EGC, early gastric cancer; NET, neuroendocrine tumor; $\mathrm{OR}$, odds ratio; $\mathrm{Cl}$, confidence interval.

\section{Declarations}

\section{Ethics approval and consent to participate}


Ethical approval for the study was obtained from the institutional review board at Samsung Medical Center. Because this study was based on a retrospective analysis of existing clinical data, the requirement for informed patient consent was waived by the institutional review board (No. 2019-09-015-001).

\section{Consent for publication}

Not applicable

\section{Availability of data and materials}

All data were anonymous and stored in coded manner in a private domain. The dataset used and analysed during the current study is available from the corresponding author on reasonable request.

\section{Competing interests}

The authors declare that tey have o competing interest.

\section{Funding}

Not applicable

\section{Author contributions}

Study concept and design: Jae J Kim.

Acquisition, analysis, or interpretation of data: Jong-In Chang and Tae Jun Kim.

Writing and Drafting of the manuscript: Jong-In Chang and Tae Jun Kim.

Critical revision of the manuscript for important intellectual content: Yang Won Min, Hyuk Lee, ByungHoon Min, Jun Haeng Lee, Poong-Lyul Rhee, Jae J Kim.

Statistical analysis: Na Young Hwang and Insuk Sohn.

Study supervision: Jae J Kim.

All authors approved the final submission.

\section{Acknowledgements}

Not applicable

\section{References}

1. Yurtlu DA, Aslan F, Ayvat P, Isik Y, Karakus N, Unsal B, et al. Propofol-Based Sedation Versus General Anesthesia for Endoscopic Submucosal Dissection. Medicine. 2016;95:e3680. 
2. Nonaka S, Kawaguchi Y, Oda I, Nakamura J, Sato C, Kinjo Y, et al. Safety and effectiveness of propofol-based monitored anesthesia care without intubation during endoscopic submucosal dissection for early gastric and esophageal cancers. Dig Endosc. 2015;27:665-73.

3. Kim JH, Nam HS, Choi CW, Kang DH, Kim HW, Park SB, et al. Risk factors associated with difficult gastric endoscopic submucosal dissection: predicting difficult ESD. Surg Endosc. 2017;31:1617-26.

4. Nishiyama N, Kobara H, Fujihara S, Koduka K, Chiyo T, Kobayashi N, et al. Endoscopic Submucosal Dissection for Neoplasia of the Greater Curvature of the Upper and Middle Stomach: J-shaped Superficial Cutting and Splashed Dissection. J Gastrointestin Liver Dis. 2019;28:397-404.

5. Kang DH, Choi CW, Kim HW, Park SB, Kim SJ, Nam HS, et al. Location characteristics of early gastric cancer treated with endoscopic submucosal dissection. Surg Endosc. 2017;31:4673-9.

6. Kiriyama S, Gotoda T, Sano H, Oda I, Nishimoto F, Hirashima T, et al. Safe and effective sedation in endoscopic submucosal dissection for early gastric cancer: a randomized comparison between propofol continuous infusion and intermittent midazolam injection. J Gastroenterol. 2010;45:831-7.

7. Wadhwa V, Issa D, Garg S, Lopez R, Sanaka MR, Vargo JJ. Similar Risk of Cardiopulmonary Adverse Events Between Propofol and Traditional Anesthesia for Gastrointestinal Endoscopy: A Systematic Review and Meta-analysis. Clin Gastroenterol Hepatol. 2017;15:194-206.

8. Park CH, Shin S, Lee SK, Lee H, Lee YC, Park JC, et al. Assessing the stability and safety of procedure during endoscopic submucosal dissection according to sedation methods: a randomized trial. PLoS One. 2015;10:e0120529.

9. Yamaguchi D, Yamaguchi N, Takeuchi Y, Yukimoto T, Ikeda K, Matsumoto K, et al. Comparison of sedation between the endoscopy room and operation room during endoscopic submucosal dissection for neoplasms in the upper gastrointestinal tract. BMC Gastroenterol. 2017;17:127.

10. Yamashita K, Shiwaku H, Ohmiya T, Shimaoka H, Okada H, Nakashima R, et al. Efficacy and safety of endoscopic submucosal dissection under general anesthesia. World J Gastrointest Endosc. 2016;8:466-71.

11. Facciorusso A, Antonino M, Di Maso M, Muscatiello N. Endoscopic submucosal dissection vs endoscopic mucosal resection for early gastric cancer: A meta-analysis. World J Gastrointest Endosc. 2014;6:555-63.

12. Choi IJ, Lee NR, Kim SG, Lee WS, Park SJ, Kim JJ, et al. Short-Term Outcomes of Endoscopic Submucosal Dissection in Patients with Early Gastric Cancer: A Prospective Multicenter Cohort Study. Gut Liver. 2016;10:739-48.

13. Yoo JH, Shin SJ, Lee KM, Choi JM, Wi JO, Kim DH, et al. Risk factors for perforations associated with endoscopic submucosal dissection in gastric lesions: emphasis on perforation type. Surg Endosc. 2012;26:2456-64.

14. Oda I, Gotoda T, Hamanaka H, Eguchi T, Saito Y, Matsuda T, et al. Endoscopic submucosal dissection for early gastric cancer: Technical feasibility, operation time and complications from a large consecutive series. Dig Endosc. 2005;17:54-8. 
15. Mannen K, Tsunada S, Hara M, Yamaguchi K, Sakata Y, Fujise T, et al. Risk factors for complications of endoscopic submucosal dissection in gastric tumors: analysis of 478 lesions. J Gastroenterol. 2010;45:30-6.

16. Toyokawa T, Inaba T, Omote S, Okamoto A, Miyasaka R, Watanabe K, et al. Risk factors for perforation and delayed bleeding associated with endoscopic submucosal dissection for early gastric neoplasms: analysis of 1123 lesions. J Gastroenterol Hepatol. 2012;27:907-12.

17. Song BG, Min YW, Cha RR, Lee H, Min BH, Lee JH, et al. Endoscopic submucosal dissection under general anesthesia for superficial esophageal squamous cell carcinoma is associated with better clinical outcomes. BMC Gastroenterol. 2018;18:80.

18. Boulos NM, Burton BN, Carter D, Marmor RA, Gabriel RA. Monitored Anesthesia Care Is Associated With a Decrease in Morbidity After Endovascular Angioplasty in Aortoiliac Disease. J Cardiothorac Vasc Anesth. 2020;34:2440-5.

\section{Figures}

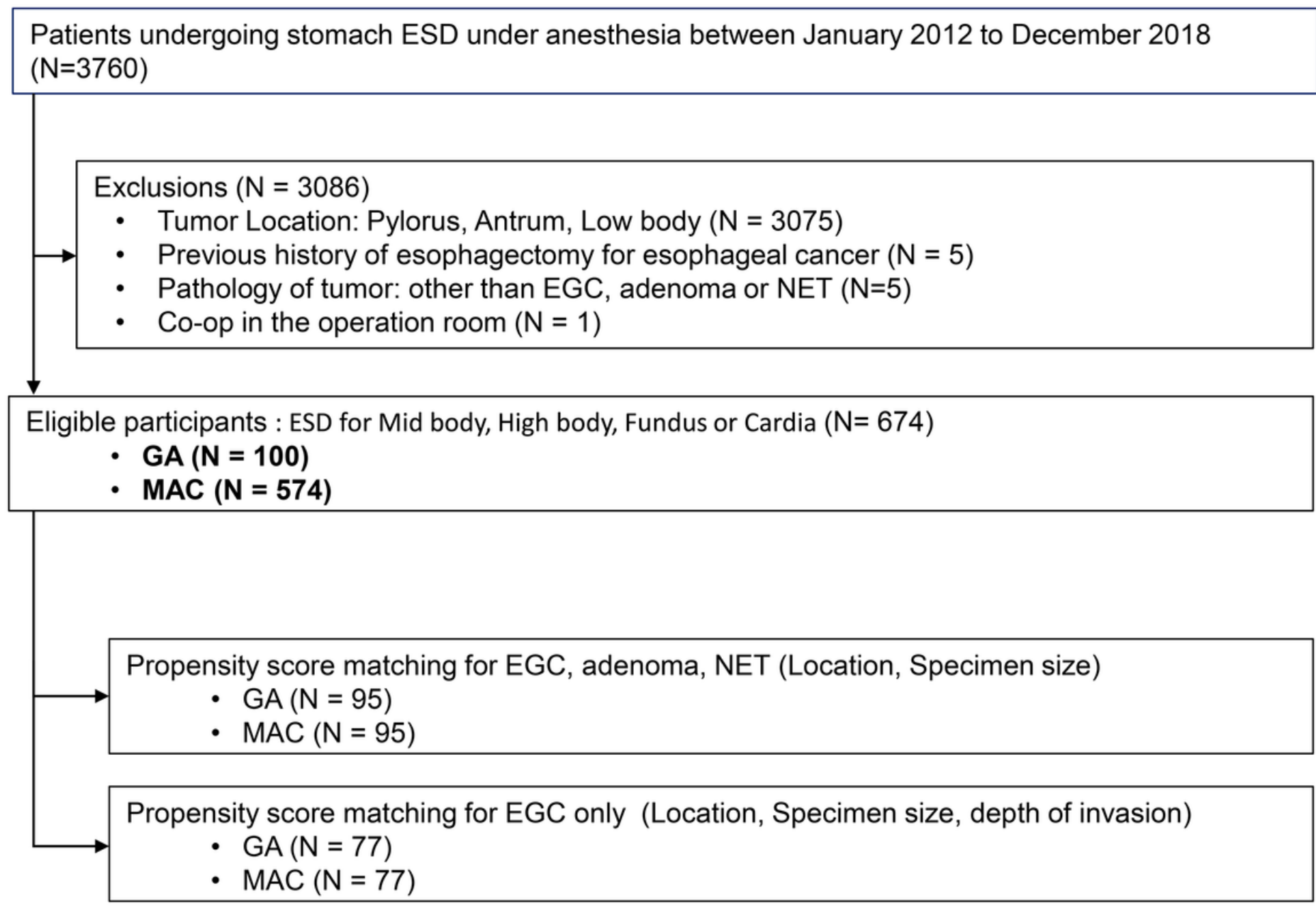

\section{Figure 1}


Flow diagram of study patients.

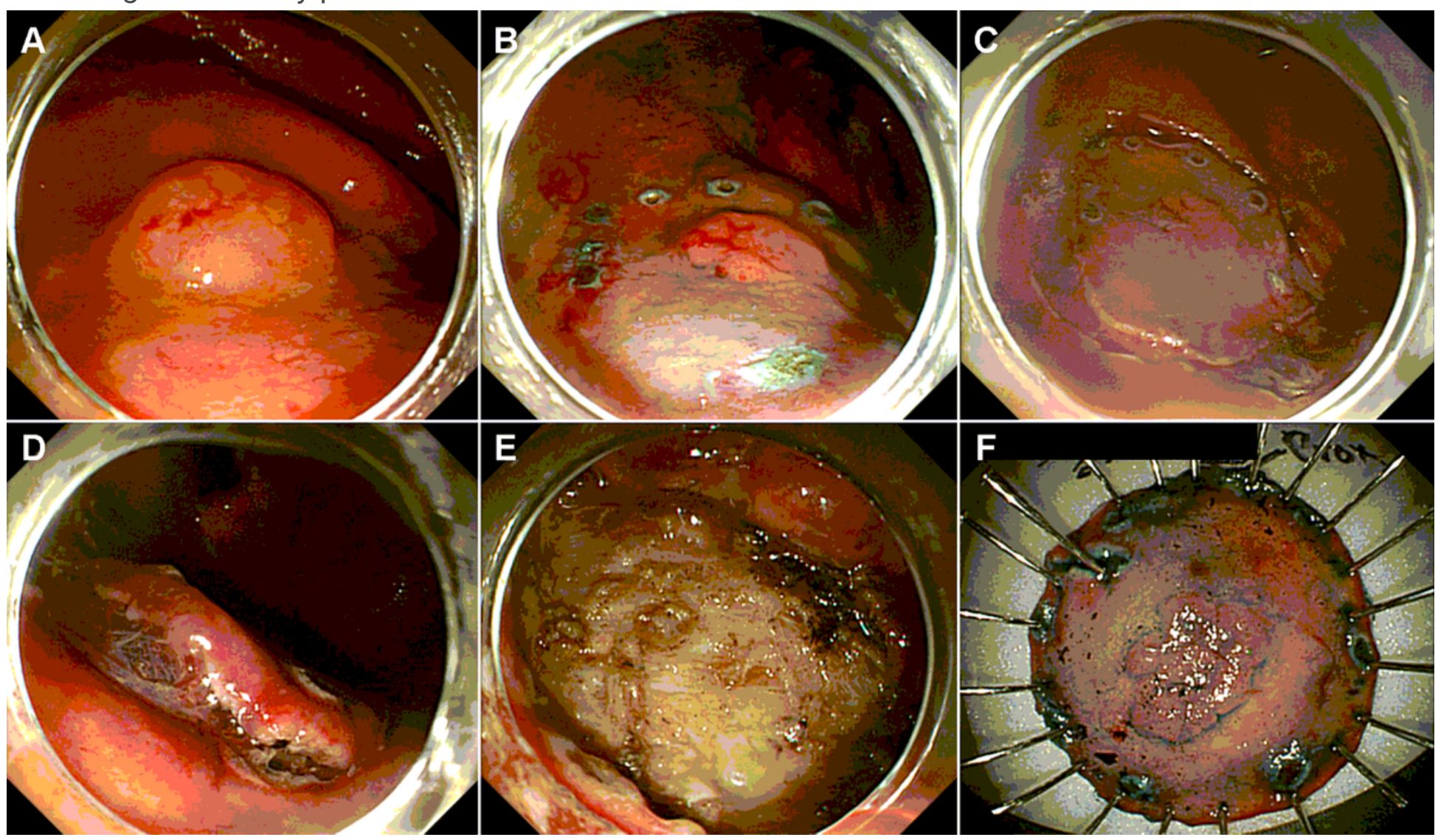

\section{Figure 2}

Endoscopic submucosal dissection procedure; (A) On the posterior wall of high body, a $1.2 \mathrm{~cm}$ sized flat elevated mucosal lesion was noticed. (B) After indigo carmine solution was sprayed for visualization of the lesion, marking around the lesion with needle knife was performed. (C) Circumferential mucosal cutting was performed with needle knife. (D) After Submucosal injection with mixed fluid of normal saline, epinephrine, indigo carmine and glycerol, dissection of submucosal layer with IT2 was performed. (E) A procedure induced artificial ulcer was seen. $(F)$ The resected specimen was fixed on a board for pathologic examination.

\section{Supplementary Files}

This is a list of supplementary files associated with this preprint. Click to download.

- Additionalfile1BMCgastroenterology0811.docx 